\title{
Radial velocity variations in pulsating Ap stars ${ }^{\star}$
}

\author{
V. 10 Aquilae \\ A. P. Hatzes ${ }^{1}$ and D. E. Mkrtichian ${ }^{2,3}$ \\ 1 Thüringer Landessternwarte Tautenburg, 07778 Tautenburg, Germany \\ e-mail: artie@tls-tautenburg.de \\ 2 Astrophysical Research Center for the Structure and Evolution of the Cosmos, Sejong University, Seoul 143-747, Korea \\ 3 Astronomical Observatory, Odessa National University, Schevchenko Park, Odessa 65014, Ukraine \\ Received 8 August 2003 / Accepted 5 August 2004

\begin{abstract}
We have used precise stellar radial velocities to study the pulsational motion of the rapidly oscillating Ap star 10 Aql. Observations were made on three separate nights using the high resolution spectrograph of the Harlan J. Smith $2.7 \mathrm{~m}$ telescope at McDonald Observatory. A high radial velocity precision was achieved by using an iodine gas absorption cell. The integrated radial velocity measurements using the full wavelength region covered by iodine absorption lines (5000-6300 $\AA$ ) failed to detect any coherent pulsational velocity variations to a level of $2.5-5 \mathrm{~m} \mathrm{~s}^{-1}$. An analysis over a much narrower wavelength range revealed that pulsational radial velocity variations are indeed present in $10 \mathrm{Aql}$, but only in 5 spectral lines. The amplitude of these variations ranged from about $100 \mathrm{~m} \mathrm{~s}^{-1}$ to as high as $398 \mathrm{~m} \mathrm{~s}^{-1}$ for an unidentified feature at $\lambda 5471.40 \AA$ A. Other spectral features showing pulsational radial velocity variations are tentatively identified as Sm II and Tm II.
\end{abstract}

Key words. stars: chemically peculiar - stars: oscillations - stars: individual: 10 Aql

\section{Introduction}

The rapidly oscillating Ap (roAp) stars are a pulsating subclass of the chemically peculiar Ap stars that exhibit p-mode oscillations with periods of 5 to $16 \mathrm{~min}$. The multi-mode nature of the roAp oscillations make these objects highly amenable to asteroseismic studies. Many roAp stars, like their non-oscillating Ap counterparts, exhibit strong dipole magnetic fields and it is largely accepted that the pulsational axis is aligned with the magnetic axis rather than the rotation axis of the star. This oblique rotator model, first proposed by Kurtz (1982) has been able to explain most of the observed photometric variations of roAp stars. The asteroseismic study of roAp stars offers us a unique opportunity to study how large magnetic fields can affect both the internal structure and outer atmosphere of stars.

In the past the study of pulsations in roAp stars largely relied on photometric measurements. In recent years radial velocity (RV) measurements have been applied to these objects with surprising results. Baldry et al. (1998) made RV measurements for $\alpha$ Cir using narrow wavelength bands (width $\approx 10 \AA$ ) and found that the pulsational amplitude in individual bands could range from $100-1000 \mathrm{~m} \mathrm{~s}^{-1}$. At the same time Kanaan \& Hatzes (1998) showed that individual spectral lines in $\gamma$ Equ could show such large differences in amplitudes. Subsequent time resolved studies established that the largest

\footnotetext{
* Based on observations collected at McDonald Observatory.
}

pulsational amplitudes seemed to come from spectral lines of rare earth elements (REE) such as Nd and Pr (Savanov et al. 1999; Khochukhov \& Ryabchikova 2001ab; Balona \& Zima 2002). However, most of these studies were based on data covering a small wavelength range or without techniques for precise $\left(\sigma<50 \mathrm{~m} \mathrm{~s}^{-1}\right)$ stellar RV measurements. RV studies of roAp stars using a broad wavelength range and precise radial velocity measurements using a simultaneous wavelength calibration have only been done for a few roAp stars.

Phase variations have also been observed in roAp stars. Baldry et al. (1998) found that approximately 15-20\% of the spectral bands in $\alpha$ Cir were almost 180 degrees out-of-phase. Khochukhov \& Ryabchikova (2001a) found pulsational phase shifts between the singly and doubly ionized REE in $\gamma$ Equ. More recently, Kurtz et al. (2003) found that the pulsational phases were constant in $\mathrm{H} \alpha$ for HD 166473, yet were variable in Pr II and III lines which presumably are formed in the upper atmosphere. The pulsational phase for Pr varied linearly with the fraction of the central intensity at which the RV was measured. This was interpreted as evidence for a standing wave in the atmosphere where $\mathrm{H} \alpha$ was formed, and a running wave in the upper atmosphere where Pr was formed.

Mkrtichian et al. (2003) found that lines of Nd III in 33 Lib oscillate approximately $180^{\circ}$ out of phase with $\mathrm{Nd}$ II lines that are formed in a different layer of the atmosphere. This was interpreted as a standing wave in the stellar atmosphere with a 
radial node situated in the upper layers. Evidence for a second node was inferred in layers of the atmosphere close to continuum formation level based on phase jumps of the RV variations for weak Fe spectral lines. Clearly, to derive the maximum information about the oscillations in roAp stars one needs to study both the phase and amplitude variations of individual lines from a variety of atomic species. This naturally requires a broad wavelength coverage.

We have been using precise stellar radial velocity measurements spanning a broad wavelength range (5000-6300 $)$ to study the oscillations in roAp stars for the past several years. This program has already detected pulsational RV variations in 5 known roAp stars: $\gamma$ Equ (Kanaan \& Hatzes 1998), HR 1217 and HD 134214 (Hatzes et al. 1999a), 33 Lib (Hatzes et al. 1999b; Mkrtichian et al. 2003), and HD 122970 (Hatzes et al. 2000). These studies has also established $\beta \mathrm{CrB}$ to be a ultra-low amplitude roAp star (Hatzes \& Mkrtichian 2004). Here we present the results of a search for RV variations in the roAp star 10 Aql.

10 Aql (HR 7167; HD 176232) is an F0 SrEu star (Hoffleit 1982) with a surface magnetic field strength showing reversing polarity over timescales of years Babcock (1958). The roAp status of $10 \mathrm{Aql}$ was reported by Heller \& Kramer (1988) and it has one of the lowest photometric amplitudes for these class of objects. In a subsequent analysis based on $26 \mathrm{~h}$ of photometric data Heller \& Kramer (1990) found the presence of 3 pulsation periods: a dominant mode $f_{1}=1.436 \mathrm{mHz}$ $\left(=124.07\right.$ cycles $\mathrm{d}^{-1}$, period $\left.=11.60 \mathrm{~min}\right), \Delta B=0.44 \mathrm{mmag}$, $f_{2}=1.385 \mathrm{mHz}\left(119.66\right.$ cycles d $^{-1}$, period $\left.=12.03 \mathrm{~min}\right)$, $\Delta B=0.33 \mathrm{mmag}$, and $f_{3}=1.239 \mathrm{mHz}\left(107.07\right.$ cycles $\mathrm{d}^{-1}$, period $=13.45 \mathrm{~min}), \Delta B=0.28 \mathrm{mmag}$. These frequencies did not appear to be equally spaced contrary to what is expected from p-mode oscillations, but Heller \& Kramer (1990) did note that the amplitudes were very close to the noise level and this could affect the selection of frequencies. A choice of nearby aliases would make the frequencies more evenly spaced.

Recently, Kochukhov et al. (2002) measured radial velocities for $10 \mathrm{Aql}$ for a total of $7.7 \mathrm{~h}$ spanning 2 nights using the Gecko spectrograph of the Canada-France-Hawaii Telescope. They failed to detect RV variations in most of the spectral lines in their wavelength interval (6105-6190 ̊). They did, however, find pulsational RV variations in 3 spectral lines: Nd III $\lambda 6145 \AA$, Eu II $\lambda 6173 \AA$, and Gd II $\lambda 6180 \AA$. Pulsational amplitudes ranged from 35 to $135 \mathrm{~m} \mathrm{~s}^{-1}$. Most periods were consistent, to within the errors, of the dominant mode found by Heller \& Kramer (1990). The false alarm probability (FAP) for the detections of these signals ranged from 0.005 to 0.08 .

10 Aql shows radial velocity variations in the fewest spectral lines of all known roAp stars. The observations of Kochukhov et al. (2002) were made over a narrow wavelength range $(\approx 85 \AA)$, so it is not known how many more spectral lines show RV variations. Furthermore, these RV measurements were made without a simultaneous wavelength calibration. The Gecko spectrograph used for these measurements can show short term instrumental shifts on some nights that are as large as $300 \mathrm{~m} \mathrm{~s}^{-1}$ (Walker et al. 2003). These were based on Th-Ar calibration lamps taken every 20 or $40 \mathrm{~min}$. The amplitude of instrumental shifts on timescales of minutes (pertinent
Table 1. Journal of observations.

\begin{tabular}{cccc}
\hline \hline Date & UT & JD-2400000 & No. of obs. \\
\hline 26 July 1997 & $6: 14-10: 37$ & 50655.762 & 153 \\
11 August 1998 & $2: 47-5: 53$ & 51036.636 & 100 \\
13 August 1998 & $3: 04-7: 10$ & 51038.632 & 145 \\
\hline
\end{tabular}

for roAp stars) has not yet been quantified. Use of simultaneous wavelength calibration will minimize such instrumental shifts and thus make RV detections more certain. Here we present rapid, precise stellar RV measurements for 10 Aql taken with a self-calibrated iodine absorption cell.

\section{Observations}

Observations of 10 Aql were made for 4.4 consecutive $\mathrm{h}$ on 26 July 1997, 3.1 h on 11 Aug. 1998 and 4.1 h 13 Aug. 1998. High resolution spectra were acquired using the 2 dcoude spectrograph (Tull et al. 1995) of the 2.7-m Harlan J. Smith telescope at McDonald Observatory. The instrument was configured to achieve a resolving power, $R(=\lambda / \Delta \lambda)=60000$ with a wavelength coverage of 4700-7000 $\mathrm{A}$. Exposure times were set at $60 \mathrm{~s}$ which was a reasonable compromise between maximizing the signal-to-noise ratio of individual observations, yet minimizing the amount of pulsational phase smearing. The typical signal-to-noise ratios (per pixel) were $\approx 70$.

To minimize the dead time between exposures the Tektronix $2048 \times 2048 \mathrm{CCD}$ detector was framed to read a subset of the full array (normally the detector covers a 4000-10 $000 \AA$ range). The detector was also binned by a factor of two perpendicular to the direction of dispersion. The total deadtime, including storage of the observation, was about $15 \mathrm{~s}$. Table 1 summarizes the journal of the observations.

Precise stellar radial velocity measurements were made using a velocity metric provided by an iodine $\left(\mathrm{I}_{2}\right)$ absorption cell. Molecular iodine has useful absorption lines in the wavelength interval 5000-6300 (14 spectral orders), although redward of $6000 \AA$ these lines become weak and this degrades the RV precision. The sub-framing of the CCD was chosen so as to cover the full wavelength region spanned by $\mathrm{I}_{2}$ absorption lines, as well as the hydrogen $\mathrm{H} \alpha$ and $\mathrm{H} \beta$ lines. The radial velocity reduction procedure requires a high signal-to-noise spectrum of the star taken without the cell in the light path. For this an exposure of several minutes was taken.

\section{Results}

\subsection{Integrated $R V$ variations}

The data were first used to compute a "mean" RV value using all of the spectral lines covered by the iodine absorption lines. It is well known that the individual lines of roAp stars can show different RV variations and that a line-by-line RV analysis may be more appropriate, but the RV error measurement for an individual line can be large which means only high amplitude RV variations can be detected. Averaging the RV variations of all spectral lines results in a very high precision measurement which may detect ultra-low amplitude, coherent pulsation 


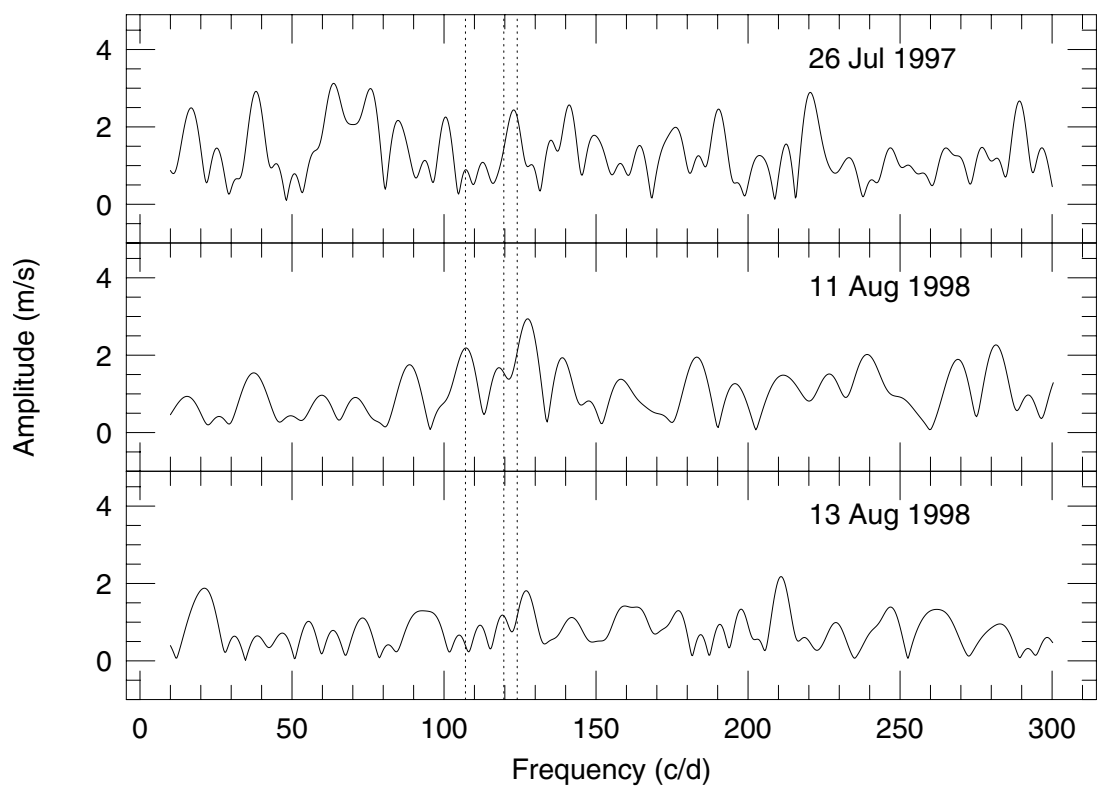

Fig. 1. The discrete Fourier transform of the integrated RV measurements for each of the nights of our observations. The vertical dashed lines mark the frequencies of the known photometric modes.

modes. Furthermore, the integrated RV variations can be more directly compared to photometric variations which are also integrated measurements. A recent analysis of the Ap star $\beta \mathrm{CrB}$ (Hatzes \& Mkrtichian 2004) showed periodic low amplitude $\mathrm{RV}$ variations $\left(K=3.5 \mathrm{~m} \mathrm{~s}^{-1}\right)$ that were first detected in the integrated RV measurements, but only in one spectral line covering the 5000-6300 ̊ region. Mkrtichian \& Hatzes (2005) also using "broad-band" RV measurements (integrated RV measurements from lines spanning $\approx 100 \AA$ ) as well single-line measurements of a $\mathrm{Nd}$ feature discovered two additional pulsation modes in the roAp star HR 1217. These modes were confirmed by RV measurements from a single spectral line of $\mathrm{Nd}$. Clearly, much can be learned from such integrated RV measurements and to exploit fully the information content of spectra one should examine both the integrated and line-by-line $\mathrm{RV}$ variations.

Each spectral order was divided into 30 spectral chunks and the RV calculated in each chunk. The achieve the highest RV precision the spatial and temporal variations of the instrumental profile must also be modeled as determined using the iodine absorption lines. This was done largely following the procedure outlined in Valenti et al. (1995) and Butler et al. (1996). The individual RV from all spectral chunks were then combined to form a mean RV value for that observation. This measurement of the RV is consistent with those obtained by cross-correlation techniques. This is well established procedure that has demonstrated an RV precision of better than $3 \mathrm{~m} \mathrm{~s}^{-1}$ on narrow-lined solar-type stars. Table 2 shows the rms scatter of the integrated RV measurements for $10 \mathrm{Aql}$ for each of the nights. The higher rms scatter for the night of 26 July 1997 is due to the lower signal-to-noise of that data because of poorer observing conditions.

Figure 1 shows the discrete Fourier transform of the integrated RV measurements for each night. The are no pulsation modes present with amplitudes greater than about
Table 2. The rms scatter for the integrated RV measurements and detection limit of an 11.6 min input sine-wave at a false alarm probability of 0.01 .

\begin{tabular}{ccc}
\hline \hline Data Set & $\begin{array}{c}\sigma_{\mathrm{RV}} \\
\mathrm{m} \mathrm{s}^{-1}\end{array}$ & $\begin{array}{c}\text { Detection Limit } \\
\mathrm{m} \mathrm{s}^{-1}\end{array}$ \\
\hline 26 July 1997 & 8.3 & 5.5 \\
11 August 1998 & 4.6 & 2.5 \\
13 August 1998 & 4.8 & 2.5 \\
\hline
\end{tabular}

2-3 $\mathrm{m} \mathrm{s}^{-1}$. Monte Carlo simulations were performed to determine the detection limit of coherent pulsational RV variations in these integrated RV measurements. An input sine wave with the frequency of the primary pulsation mode $\left(f_{1}=\right.$ $124.07 \mathrm{c} \mathrm{d}^{-1}=1.43 \mathrm{mHz}$ ) was sampled in the same manner as the data. Random noise was added with a standard deviation consistent with the rms scatter of the RV variations. The amplitude of the input sine wave was then varied until a Lomb-Scargle periodogram (Lomb 1976; Scargle 1982) yielded power at the input frequency at a false alarm probability of $1 \%$. These simulations show that we would have detected a known pulsation mode at an amplitude as small as $5 \mathrm{~m} \mathrm{~s}^{-1}$ on 26 July 1997 and an amplitude as small as $2.5 \mathrm{~m} \mathrm{~s}^{-1}$ on 11 and 13 Aug. 1998.

\section{2. $R V$ variations of individual lines}

Kanaan \& Hatzes (1998) first demonstrated that the pulsational RV amplitude in roAp stars can differ by factors of ten or more between different spectral lines. Furthermore, Kochukhov et al. (2002) reported RV variations in 10 Aql in only a few spectral features. If this is the case then some spectral lines $10 \mathrm{Aql}$ could have a measurable pulsational amplitude that would be averaged below detection limits when computing the radial velocity over the full wavelength region that includes many lines. 


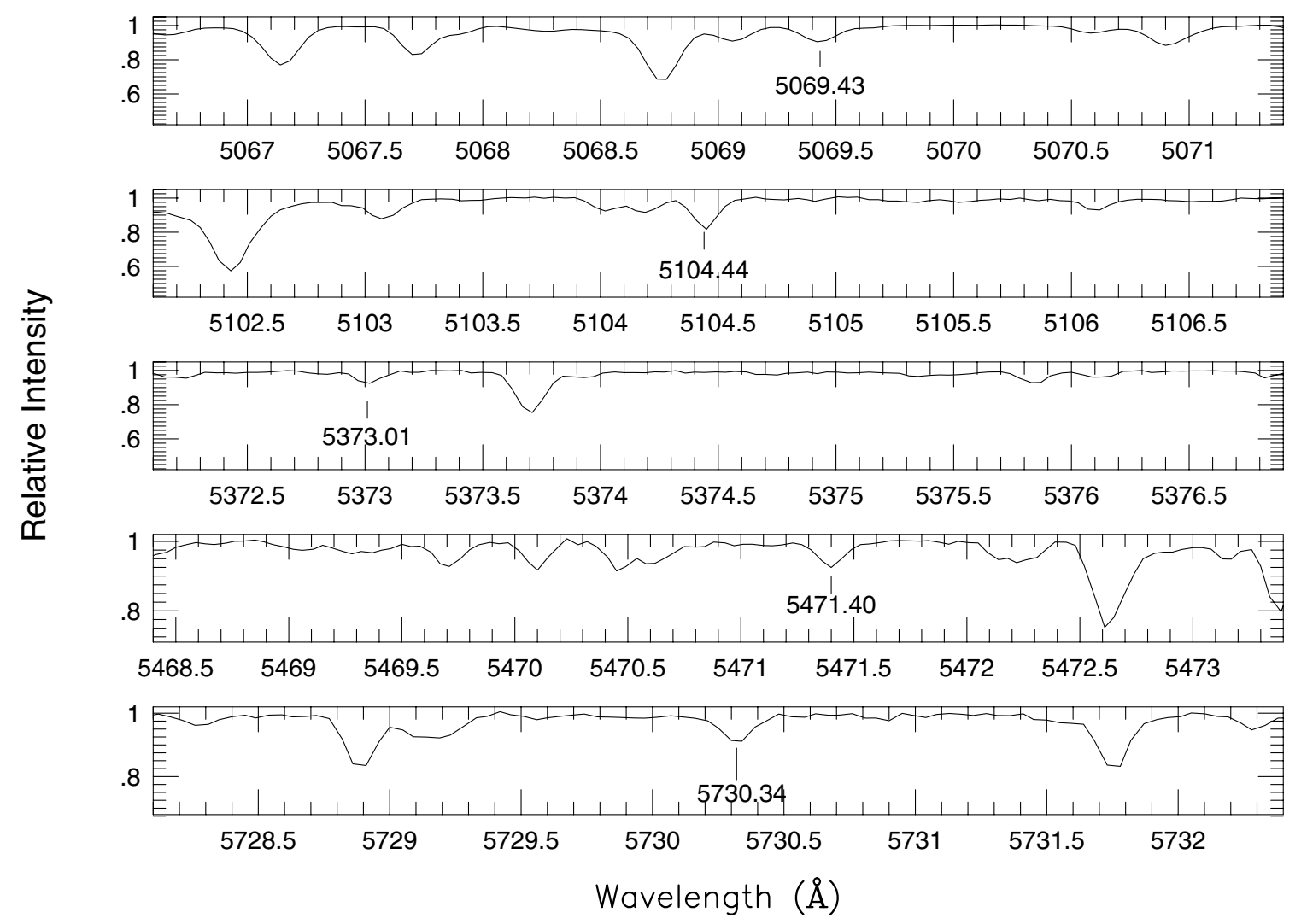

Fig. 2. The spectral region of $10 \mathrm{Aql}$ near the high amplitude RV lines which are marked by vertical lines.

Table 3. Spectral line identifications.

\begin{tabular}{cl}
\hline \hline Wavelength $(\AA)$ & Identification \\
\hline$\lambda 5069.43$ & $\lambda 5069.47 \mathrm{Sm}$ II \\
& $\lambda 5069.42 \mathrm{Fe}$ I \\
$\lambda 5104.44$ & $\lambda 5104.48 \mathrm{Sm}$ II \\
& $\lambda 5104.44 \mathrm{Fe}$ II \\
$\lambda 5373.01$ & $\lambda 5373.01 \mathrm{Tm}$ II \\
$\lambda 5471.40$ & Unidentified \\
$\lambda 5730.34$ & Unidentified \\
\hline
\end{tabular}

To expedite the analysis the RV was first determined on the individual chunks that spanned about $4 \AA$ and which included just a few spectral lines. If a periodogram of the RV measurements produced significant power at a pulsational frequency $(\mathrm{FAP} \approx 0.02)$, then the wavelength window was reduced to include only one spectral line. This was repeated for every spectral line in the window so that the spectral feature(s) responsible for the RV variations was (were) isolated. This procedure ensured that we would find lines with statistically significant variations which minimizes the risk of trying to interpret signals that are due to noise.

Our analysis yielded only five spectral lines which exhibited significant evidence for RV variations at one of the photometric frequencies. These lines are listed in Table 3 along with the measured wavelength (corrected for the Earth's barycentric motion and the absolute RV of $10 \mathrm{Aql}$ ) and the tentative identification of the feature. The identifications were based on the coincidence of the wavelengths of our spectral features to wavelengths and intensity of those calculated using the VALD (Piskunov et al. 1995) "extract stellar" option and the $T_{\text {eff }}=$ $7500, \log g=4.0$ model atmosphere. This provided the closest match to the stellar parameters for $10 \mathrm{Aql}$ (Ryabchikova et al. 2000). The wavelengths of the DREAM (Biemont et al. 2003) database and the line-list of Przybylski's star (Cowley et al. 1998) were also used. Two lines at $\lambda 5471.40 \AA$ and $\lambda 5730.34 \AA$ are unidentified in the spectrum of $10 \mathrm{Aql}$. The same lines were also found unidentified in the spectrum of Przybylski's star. Figure 2 shows the spectral regions of these high amplitude spectral lines.

Figure 3 shows the discrete Fourier transform (amplitude is in $\mathrm{m} \mathrm{s}^{-1}$ ) for these five spectral features. The Fourier transform for a single spectral line on each night is shown horizontally. Vertical dashed lines indicate the location of the published photometric frequencies.

The dominant photometric mode, $f_{1}\left(=124.07 \mathrm{c} \mathrm{d}^{-1}=\right.$ $1.436 \mathrm{mHz}$ ) is the one found in nearly all of the RV variations for individual spectral lines. There is only one instance where another mode $\left(f_{3}=107 \mathrm{c} \mathrm{d}^{-1}=1.239 \mathrm{mHz}\right)$ may be present and that is for $\lambda 5069 \AA$ on the night of $26 \mathrm{Jul} 1997$. Table 4 lists the detected period, amplitude, and phase for the 5 spectral lines for each of the nights. The period was taken as the highest peak in the periodogram nearest a photometric frequency. In most instances this peak also coincided with the highest peak in the frequency interval that was examined. The amplitudes listed in Table 4 result from a least squares sine fit to the data using the period found in our Fourier analysis. 


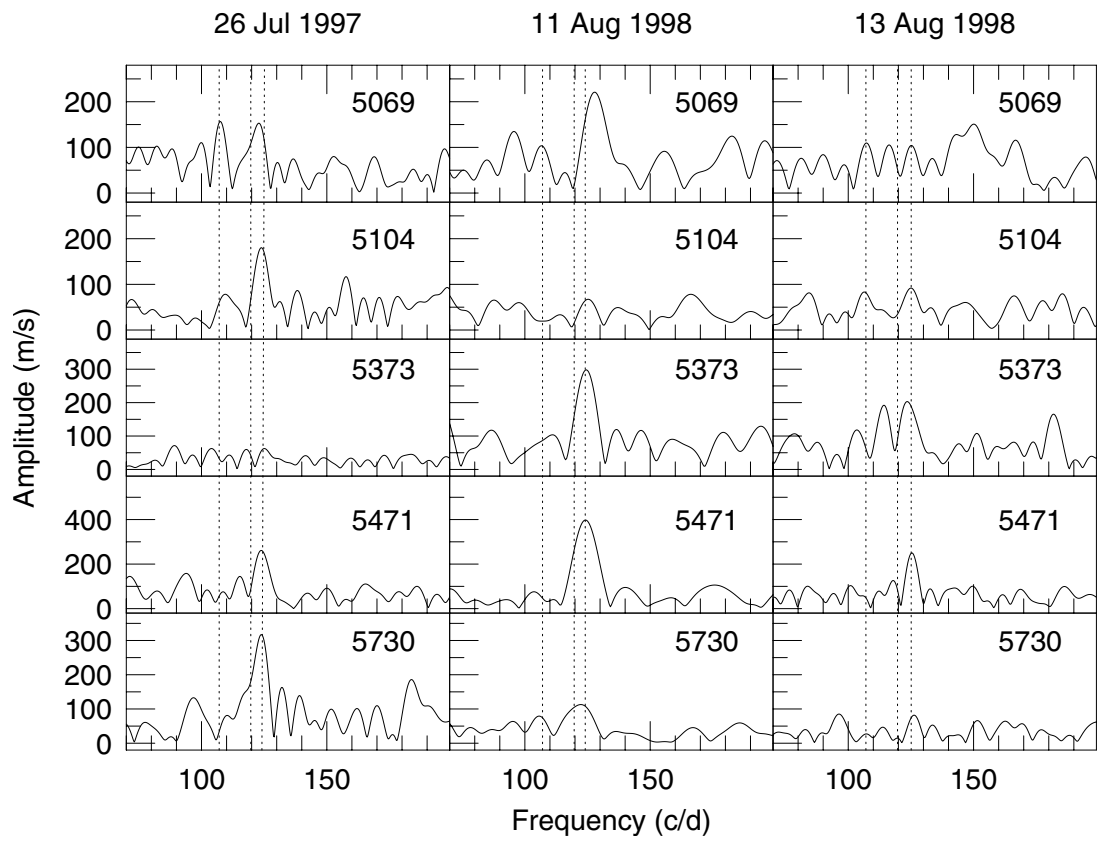

Fig. 3. The discrete Fourier transform for the spectral lines showing evidence for pulsational RV variations for the three nights of our observations. Each night is shown in vertical panels. The number in the upper right corner of each panel is the wavelength of the spectral line in $\AA$.

Table 4. Pulsational amplitude and phases for spectral lines in 10 Aql. False alarm probabilities (FAP) were computed assuming no a priori knowledge of the presence of possible frequencies. The FAP values in parentheses are for a pre-selected (photometric) frequency.

\begin{tabular}{c|ccrcc}
\hline \hline & \multicolumn{5}{|c}{ 26 Jul. 1997 } \\
\hline Line $(\AA)$ & $v\left(\mathrm{c} \mathrm{d}^{-1}\right)$ & $P(\mathrm{~min})$ & $K\left(\mathrm{~m} \mathrm{~s}^{-1}\right)$ & $\phi$ & FAP \\
\hline$\lambda 5069.43$ & 107.5 & $13.39 \pm 0.67$ & $153.7 \pm 42.3$ & $0.37 \pm 0.044$ & $0.12(0.0015)$ \\
$\lambda 5069.43$ & 122.9 & $11.72 \pm 0.51$ & $152.0 \pm 42.5$ & $0.37 \pm 0.045$ & $0.14(0.002)$ \\
$\lambda 5104.44$ & 124.0 & $11.61 \pm 0.29$ & $175.1 \pm 27.8$ & $0.38 \pm 0.031$ & $1.5 \times 10^{-3}$ \\
$\lambda 5373.01$ & 125.1 & $11.51 \pm 0.53$ & $63.0 \pm 21.0$ & $0.71 \pm 0.053$ & $0.51(0.016)$ \\
$\lambda 5471.40$ & 123.9 & $11.62 \pm 0.38$ & $262.7 \pm 47.6$ & $0.60 \pm 0.028$ & $<10^{-5}$ \\
$\lambda 5730.34$ & 124.0 & $11.61 \pm 0.29$ & $236.7 \pm 45.0$ & $0.60 \pm 0.026$ & $<10^{-5}$ \\
\hline & \multicolumn{5}{|c}{11 Aug. 1998} \\
\hline \hline$\lambda 5069.43$ & 127.7 & $11.28 \pm 0.38$ & $215.5 \pm 40.8$ & $0.32 \pm 0.030$ & $4.4 \times 10^{-4}$ \\
$\lambda 5104.44$ & 125.2 & $11.50 \pm 0.91$ & $68.5 \pm 27.8$ & $0.32 \pm 0.062$ & $0.85(0.075)$ \\
$\lambda 5373.01$ & 124.6 & $11.56 \pm 0.42$ & $301.3 \pm 63.1$ & $0.55 \pm 0.033$ & $2.7 \times 10^{-3}$ \\
$\lambda 5471.40$ & 124.8 & $11.54 \pm 0.18$ & $398.0 \pm 43.9$ & $0.55 \pm 0.016$ & $<10^{-5}$ \\
$\lambda 5730.34$ & 122.1 & $11.79 \pm 0.59$ & $112.6 \pm 30.6$ & $0.64 \pm 0.042$ & $0.19(0.01)$ \\
\hline & \multicolumn{5}{|c}{13 Aug. 1998} \\
\hline \hline$\lambda 5069.43$ & 125.1 & $11.51 \pm 0.72$ & $99.6 \pm 47.6$ & $0.37 \pm 0.079$ & $0.96(0.08)$ \\
$\lambda 5104.44$ & 125.1 & $11.51 \pm 0.56$ & $92.0 \pm 30.0$ & $0.31 \pm 0.075$ & $0.41(0.012)$ \\
$\lambda 5373.01$ & 122.3 & $11.77 \pm 0.90$ & $121.0 \pm 41.2$ & $0.60 \pm 0.049$ & $0.55(0.014)$ \\
$\lambda 5471.40$ & 125.2 & $11.50 \pm 0.32$ & $199.0 \pm 40.0$ & $0.54 \pm 0.032$ & $2.7 \times 10^{-4}$ \\
$\lambda 5730.34$ & 126.3 & $11.40 \pm 0.40$ & $82.9 \pm 24.4$ & $0.55 \pm 0.046$ & $0.21(0.004)$ \\
\hline & \multicolumn{5}{c}{} \\
\hline
\end{tabular}

There are large amplitude variations between the different spectral lines and for the same line, but on different nights. The largest amplitude is found in an unidentified spectral feature at $\lambda 5471.40 \AA$. The highest amplitude for this line occurred on 11 Aug. 1998 with a semi-amplitude $(K)=398 \pm 43 \mathrm{~m} \mathrm{~s}^{-1}$, but this dropped to $199 \pm 40 \mathrm{~m} \mathrm{~s}^{-1}$ on the successive night. Figure 4 shows the RV variations of $\lambda 5471.40 \AA$ feature for the night where it had the highest amplitude and Fig. 5 shows the same RV variations phased to a period of $11.6 \mathrm{~min}$. 


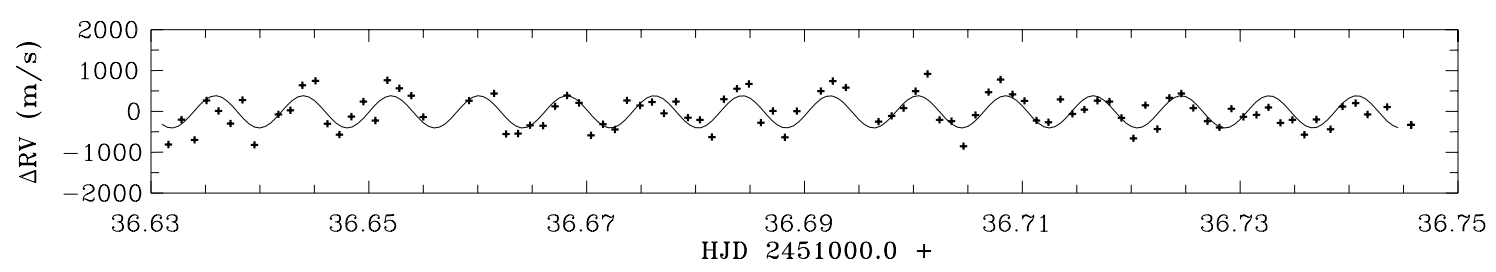

Fig. 4. The radial velocity variations for the $5471.40 \AA$ A feature on the night of 11 Aug. 1998 .

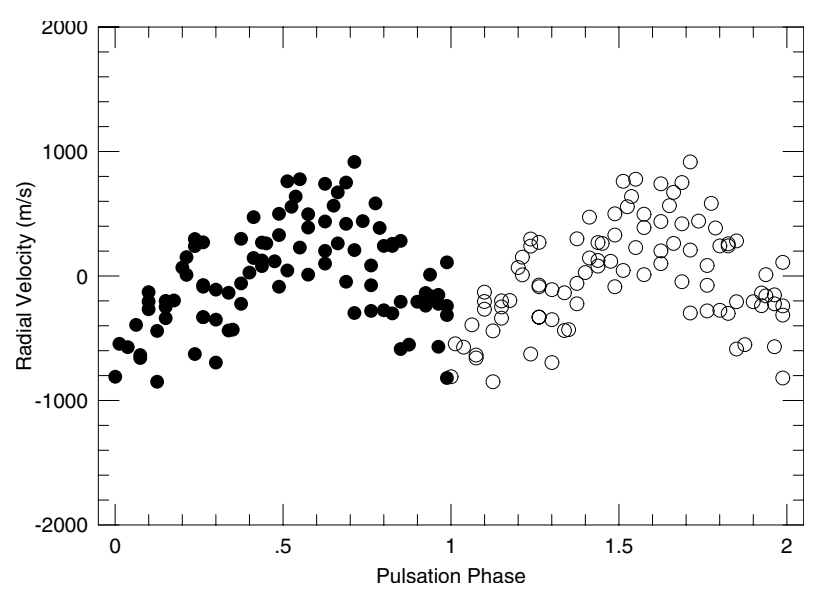

Fig. 5. The radial velocity variations for the $\lambda 5471.40 \AA$ feature on the night of 11 Aug. 1998 phased to the pulsation period of $11.54 \mathrm{~min}$ (filled circles). The values are repeated for the second cycle (open circles).

For consistency, the phases in Table 4 were reckoned using the same frequency, $f_{1}$, derived from the photometry. Most of the frequencies in Table 3 differ slightly to that of the photometric mode $f_{1}$ and these may introduce artificial phase shifts. (The only exception was the $13.39 \min$ period $\left(v=107.5 \mathrm{c} \mathrm{d}^{-1}\right)$ of the $15069 \AA$ feature.) Using a single frequency minimizes this. Because the data span such a large time difference and the pulsation periods are not known to a high enough accuracy to use the same zero point, the phases in Table 3 were reckoned using a different starting time. Comparisons of the pulsational phases between different lines should only be made between phases on a given night. The zero points used were $T_{0}=2450655.7629$ (26 July 1997), 2451036.6357 (11 August 1998), and 2451038.632 (13 August 1998). The phases listed in Table 4 correspond to the phase of minimum radial velocity.

In Table 4 one can note phase differences between different lines on different nights, but we will not comment on this further as it was done in our previous paper on $33 \mathrm{Lib}$ (Mkrtichian et al. 2003). The relatively small amount of spectral lines showing RV variations and the multiperiodicity of 10 Aql may introduce significant phase errors that are larger than the formal errors of the least-square solutions. A careful study of the phase variations in multiperiodic stars would require considerably more data.

Kochukhov et al. (2002) reported RV variability in 10 Aql in 3 spectral lines. Unfortunately, the spectral region used by Kochukhov et al. is in a region where the iodine absorption lines are too weak to give a good measurement precision $\left(\sigma \approx 150 \mathrm{~m} \mathrm{~s}^{-1}\right)$ for an individual line. A periodogram analysis was performed on the wavelength chunks containing Nd III $\lambda 6145 \AA$, Eu II $\lambda 6173 \AA$, and Gd II $\lambda 6180 \AA$. No significant RV variations were found to a level of about $100 \mathrm{~m} \mathrm{~s}^{-1}$.

\subsubsection{Statistical significance of peaks}

The statistical significance of the detected signals was estimated using a bootstrap randomization technique (Murdoch et al. 1993; Kürster et al. 1993). The measured RV values were randomly shuffled keeping the observed times fixed. A periodogram was then computed for each "random" data set. The fraction of a large number of periodograms $\left(10^{5}\right.$ in this case) having power higher than the data periodogram in the range $50<v<200 \mathrm{c} \mathrm{d}^{-1}$ represents the false alarm probability that noise would create the detected signal. This is a more rigorous means of establishing the FAP over simple use of the power in the Lomb-Scargle periodogram. The false alarm probabilities are listed in Table 4.

We note that this is a very conservative estimate of the FAP. A more liberal (and possibly more appropriate) estimate of the FAP should be made by asking what the probability is that noise will produce a peak higher than the observed peak exactly at a certain frequency. In this instance the frequency range over which the maximum power is searched should be narrowly confined to an interval spanning this known frequency, not the broad range we have considered. In this instance the FAP will be significantly less. This FAP, also determined from the Monte Carlo simulations, is listed in parentheses in Table 3 for a few of the weaker detections.

\section{Discussion}

Our radial velocity measurements of $10 \mathrm{Aql}$ along with those of Kochukhov et al. (2002) and the photometric measurements of Heller \& Kramer (1988) confirm the roAp status of 10 Aql. The RV variations found in 3 of our spectral lines had FAP $<10^{-5}$, or a factor of at least 1000 less than the FAP of the Kochukhov et al. (2002) detections. Furthermore, since our RV measurements are made with a simultaneous wavelength reference we can exclude any instrumental orgin for the RV variations. This establishes without doubt the pulsational RV variability in 10 Aql.

The dominant photometric mode, $f_{1}$, is the one present in most of our spectral lines. Our mean value for $f_{1}$ determined from the periodograms showing the strongest signal (excluding the $\lambda 5069 \AA$ feature $)$ is $123.31 \pm 0.5 \mathrm{c} \mathrm{d}^{-1}(=1.440 \pm$ $0.0058 \mathrm{mHz}$ ). The $15069 \AA$ feature on 26 July shows a peak at a significantly higher frequency of $127.6 \pm \mathrm{c} \mathrm{d}^{-1}=(1.477 \pm$ $0.05 \mathrm{mHz}$ ). Although this is consistent with the location of $f_{1}$ to within the errors, it is nearly $5 \sigma$ away from the mean value 
of $f_{1}$ determined from other spectral lines. We note that for the two other spectral lines with strong power at $f_{1}$ on this night $\left(\lambda 5373 \AA\right.$ and $\lambda 5471 \AA$ ) had a mean frequency of $128.4 \mathrm{c} \mathrm{d}^{-1}$ $(=1.446 \mathrm{mHz})$ and these data had the same sampling as for the $\lambda 5069 \AA$ A feature. One intriguing possibility is that $\lambda 5069 \AA$ on 26 July shows evidence of another mode. If we assume a frequency spacing of the $\mathrm{p}$-modes defined by the difference in frequency of the first two photometric modes, i.e. $\Delta v=f_{1}-f_{2}$, then a new mode could be located at a frequency of $1.487 \mathrm{mHz}$, tantalizing close to the detected frequency. A more detailed investigation with longer time series is needed to confirm this.

We find no strong evidence for the presence of photometric modes $f_{2}$ and $f_{3}$ in any of the 5 spectral features showing strong RV variations, with the possible exception of the $\lambda 5069 \AA$ Aine on the night of 26 July 1997. The highest peak of the RV Fourier transform is coincident with $f_{3}$ and the FAP is reasonably low ( 0.0015 , for a pre-selected frequency). The second highest peak is at frequency of about $121 \mathrm{c} \mathrm{d}^{-1}$ and could be the unresolved blend of $f_{1}$ and $f_{2}$. Again, we would be more confident of this result with more data.

All of the features in Fig. 3 show strong variations in the amplitude from night to night. The rotation period of $10 \mathrm{Aql}$ is unknown so it is not clear if these pulsation amplitude variations are due to rotational modulation.

Probable small periodic variations of equivalent widths of chromium, iron, and calcium lines with a period of 6.02 days in $10 \mathrm{Aql}$ were recently reported by Kallinger et al. (2003). If this represents the rotational period then our observations on the nights 11 Aug. and 13 Aug. 1998 may show rotational modulation of the pulsational amplitude as these were separated by 0.33 of a rotation period. However, the mean integrated relative RV of 10 Aql on these two nights agree to within $2 \mathrm{~m} \mathrm{~s}^{-1}$ which at face value excludes rotational modulation with periods of several days.

A longer rotation period also seems consistent with the magnetic field measurements of Preston (1970) which show a constant effective field strength of $H_{\mathrm{e}}=500 \pm 100$ Gauss over a 10 day span. The sparse magnetic field measurements of Babcock (1958) showed field reversals, but on time scales of years. The errors of these measurements $(\approx \pm 30 \mathrm{G})$ were about one-tenth the measured field strength, and as commented by Babcock: "The magnetic field is measured with precision and shows a reversal of polarity". These measurements imply a rotation period much greater than 10 days, and possibly of order years. Clearly, confirmation of the 6 day period in $10 \mathrm{Aql}$ is important for the interpretation of the amplitude variations in 10 Aql.

Alternative hypotheses are that the amplitude variations result from the growth and decay of the pulsational mode or close multiperiodicity. Data of a much longer time series are needed to answer this.

What distinguishes 10 Aql from the other roAp stars for which we have measured RV variations is the complete lack of RV variability for most spectral lines throughout the 5000-6300 ̊ wavelength region of 10 Aql. In fact, we can exclude the presence of integrated RV variations in $10 \mathrm{Aql}$ down to a level of $2.5 \mathrm{~m} \mathrm{~s}^{-1}$. Integrated $\mathrm{RV}$ measurements have always detected RV pulsational variations, even in the new, ultra-low amplitude roAp star $\beta$ CrB (Hatzes \& Mkrtichian 2004). (Interestingly, high amplitude RV variations were only found in one spectral line of $\beta \mathrm{CrB}$ at $\lambda 6727 \AA$, a probable $\mathrm{Ce}$ II line blended with a Cr II feature.)

Why do most spectral lines in $10 \mathrm{Aql}$ show no RV variability? The answer may lie in the results of an RV study of the roAp star 33 Lib. Mkrtichian et al. (2003) found evidence for two radial nodes in the atmosphere of $33 \mathrm{Lib}$. RV variations of the Nd III lines that are possibly formed higher in the stellar atmosphere and thus above a node oscillate approximately $180^{\circ}$ out-of-phase with respect to $\mathrm{Nd}$ II lines formed below the node. In the case of $33 \mathrm{Lib}$ the majority of spectral lines are formed far from the radial node. It may be that the radial node in $10 \mathrm{Aql}$ is in the center of the line formation region. Since the majority of spectral lines are formed in a region where there is little atmospheric motion due to pulsations, the RV amplitude should be very low and undetectable. The continuum, from where the photometric variations originate, lies in a region of the stellar atmosphere below where the spectral lines are formed, and hence away from the node. Thus the pulsational photometric amplitudes will have non-zero amplitudes. The hypothesis of the presence of a vertical atmospheric standing wave and acoustic node in line-forming region can explain the zero amplitudes of the majority of spectral lines and the very low photometric amplitudes that have been measured in 10 Aql.

We can think of two explanations for the high pulsational velocities of the spectral features in Table 4. The first assumes the presence of a horizontal node in the nonradial pulsations and that the surface distributions of the elements responsible for the high RV amplitude lines are inhomogeneous. They could be concentrated in overabundant and highly localized spots on the stellar surface (say at the magnetic poles where the pulsation amplitudes are expected to be large). In this case the measured RV comes from a small region of the star and there is less cancellation effects for non-radial modes. This results in an apparent amplification of the observed disk-integrated pulsational amplitude due to spatial filtering of nonradial pulsations (Mkrtichian 1994).

For most elements, if they are evenly distributed across the surface of $10 \mathrm{Aql}$, cancellation effects would reduce the RV amplitude to below detectable levels. This would occur in both dipole and quadropole modes if $10 \mathrm{Aql}$ is viewed from a large angle $\left(\approx 45-90^{\circ}\right)$ with respect to the pulsational (and thus magnetic) axis. The variations in amplitude from night to night could arise from rotational modulation as the spots move out of view. However, the rotational period of $10 \mathrm{Aql}$ is not known with certainty and spectral monitoring of $10 \mathrm{Aql}$ is needed to establish if the certain elements are indeed concentrated in spots on the surface.

An alternative and probably more realistic explanation is within the framework of the vertical acoustic node hypothesis (Baldry et al. 1998). This assumes that the elements of the high amplitude lines are stratified and formed at a different height in the stellar atmosphere from most spectral lines in $10 \mathrm{Aql}$. Such stratification has been found in other roAp stars (Ryabchikova et al. 2002). If these regions are sufficiently far from the radial 
node then the RV amplitude should be higher. Vertical stratification in the atmosphere of 10 Aql may cause the lines from certain atomic species to be formed at layers far above the average line forming region. There is observational evidence for such stratification inferred from the acoustic cross sections of the atmosphere of $33 \mathrm{Lib}$ using the Nd lines (Mkrtichian et al. 2003). It cannot be excluded that both horizontal and vertical node effects work in the atmospheres of roAp stars and these could be isolated and studied separately by an analysis of the RVs of individual spectral features.

Our RV analysis of the high amplitude spectral lines was able to find the dominant photometric period found by Heller \& Kramer (1988), although there are strong amplitude variations from night to night. We suspect that this is due to short-term amplitude variability of modes or close multiperiodicity. A less probable explanation that this is due to rotational modulation.

Acknowledgements. D.E.M. acknowledges his work as part of research activity of the Astrophysical Research Center for the Structure and Evolution of the Cosmos (ARCSEC) which is supported by the Korean Science and Engineering Foundation. Part of this work was done under the US Civilian Research and Development Foundation (CRDF) grant UP2-317 and a grant by the German Academic Exchange Service (DAAD). APH acknowledges the support of grant 50OW0204 from the Deutsches Zentrum für Luft- und Raumfahrt e.V. (DLR).

\section{References}

Babcock, H. W. 1958, ApJS, 3, 141

Balona, L. A., \& Zima, W. 2002, MNRAS, 336, 873

Baldry, I. K., Bedding, T. R., Viskum, M., Kjeldsen, H., \& Frandsen, S. 1998, MNRAS, 295, 33

Biemont, E. Palmeri, P., \& Quinet, P. 2003, DREAM database, www.umh.ac.be/ astro/dream.shtml, ftp: $\mathrm{ftp}: / /$ umhsp02 .umh.ac.be/pub/ftp_astro/dream/

Butler, R. P., Marcy, G. W., Williams, E., et al. 1996, PASP, 108, 500

Cowley, C. R., \& Mathys, G. 1998, A\&A, 339, 165

Hatzes, A.P., Kanaan, A., \& Mkrtichian, D. E. 1999a, Precise Stellar Radial Velocities, ed. J. B. Hearnshaw, \& C. D. Scarfe, ASP Conf. Ser., 185,166
Hatzes, A. P., Kanaan A., \& Mkrtichian, D. E. 1999b, Precise Stellar Radial Velocities, ed. J. B. Hearnshaw, \& C. D. Scarfe, ASP Conf. Ser., 185,183

Hatzes, A. P., Mkrtichian, D. E., \& Kanaan, A. 2000, Proc. NATOASI Conf. Variable Stars As Essential Astrophysical Tools, ed. C. Ibanoglu, NATO Science Ser. C., 544, 405

Hatzes, A. P., \& Mkrtichian, D. E. 2004, MNRAS, 351, 663

Heller, C. H., \& Kramer, K. S. 1988, PASP, 100, 583

Heller, C. H., \& Kramer, K. S. 1990, MNRAS, 244, 372

Hoffleit, D. 1982, Bright Star Catalogue, Fourth Edition, Yale University Observatory

Kallinger, T., Bigot, L., Kaiser, A., et al. 2003, in Asteroseismology Across the HR Diagram, ed. M. J. Thompson, M. S. Cunha, \& M. J. P. F. G Monteiro (Kluwer), in press

Kanaan, A., \& Hatzes, A. P. 1998, ApJ, 503, 848

Kochukhov, O., \& Ryabchikova, T. 2001a, A\&A, 374, 615

Kochukhov, O., \& Ryabchikova, T. 2001b, A\&A, 377, L22

Kochukhov, O., Landstreet, J. D., Ryabchikova, T., Weiss, W. W., \& Kupka, F. 2002, MNRAS, 337, L1

Kürster, M., Schmitt, J. H. M. M., Cutispoto, G., \& Dennerl, K. 1997, A\&A, 320, 831

Kurtz, D. W. 1982, MNRAS, 200, 807

Kurtz, D. W., Elkin, V. G., \& Mathys, G. 2003, 343, L5

Lomb, N. R. 1976, Ap\&SS, 39, 477

Murdoch, K. A., Hearnshaw, J. B., \& Clark, M. 1993, ApJ, 413, 349

Mkrtichian, D. E. 1994, Sol. Phys., 152, 275

Mkrtichian, D. E., Hatzes, A. P., \& Kanaan, A. 2003, MNRAS, 345, 781

Mkrtichian, D. E., \& Hatzes, A. P. 2005, A\&A, 430, 263

Piskunov, N. E., Kupka, F., Ryabchikova, T. A., Weiss, W. W., \& Jeffry, C. S. 1995, A\&AS, 112, 525

Preston, G. W. 1970, PASP, 82, 878

Ryabchikova, T. A., Savanov, I. S., Hatzes, A. P., Weiss, W. W., \& Handler, G. 2000, A\&A, 357, 981

Ryabchikova, T. N., Piskunov, N., Kochukhov, O., et al. 2002, A\&A, 384,545

Savanov, I. S., Malanushenko, V. P., \& Ryabchikova, T. A. 1999, Astron. Lett., 25, 902

Scargle, J. D. 1982, ApJ, 263, 835

Tull, R. G., MacQueen, P. J., Sneden, C., \& Lambert, D. L. 1995 , PASP, 107, 251

Valenti, J. A., Butler, R. P., \& Marcy, G. W. 1995, PASP, 107, 966

Walker, G., Sckolnik, E.,Bohlender, D., \& Yang, S. 2003, PASP, 115, 700 